\title{
STOCHASTIC OPTIMIZATION MODELS OF ACTUARIAL MATHEMATICS
}

\author{
Yu. M. Ermoliev, ${ }^{1}$ V. I. Norkin, ${ }^{2 \dagger}$ and B. V. Norkin ${ }^{2 \ddagger}$ \\ UDC 368:519.21
}

\begin{abstract}
The paper overviews stochastic optimization models of actuarial mathematics and methods for their solution from the point of view of the methodology of multicriteria stochastic programming and optimal control. The evolution of the capital of an insurance company is considered in discrete time. The main random parameters of the models are insurance payouts, i.e., the ratios of paid insurance claims to the corresponding premiums per unit time. Optimization variables are the structure of the insurance portfolio (gross premium structure) and amount of dividends. As efficiency criteria, indicators of the profitability of the insurance business are used, and, as risk indicators the ruin probability and the recourse capital necessary to prevent the ruin are taken. The goal of the optimization is to find Pareto-optimal solutions. Methods for finding these solutions are proposed.
\end{abstract}

Keywords: actuarial mathematics, risk process, ruin probability, stochastic programming, multicriteria problems, two-stage problems, probability constraints, stochastic optimal control.

\section{INTRODUCTION}

Traditional theory of optimal insurance is based on the expected utility theory [1,2]. An alternative approach is based on profitability and/or risk optimization [3-11]. As a risk indicator, ruin probability (insolvency probability) is most often used; an extensive literature [12-14] is devoted to its estimation and computing. Difficulty of the optimization problems that arise in this case can be explained by the fact that they belong to the class of nonconvex problems of stochastic programming or stochastic optimal control under probability constraints. The stochastic programming theory is intended to formalize problems of decision-making under stochastic uncertainty [15-18]. In the present paper, we will use model examples to show how one can formulate and numerically solve problems of insurance business optimization by several criteria within the limits of the paradigm of computing stochastic programming [19].

Unlike the classical Kramer-Lundberg model, in the paper we will consider the process of stochastic evolution of the capital of an insurance company in discrete time, which is justified by discrete time (quarter, annual) reporting of the companies on the results of their activity. The main source of randomness in insurance is insurance claims, which appear at random instants of time and are of random size. In the considered models with discrete time, the main random variable is insurance payouts: payment-to-premium ratio per unit time (year, quarter). The dynamics of making optimal decisions in these models is reflected in two- and multi-stage structure of stochastic programming models, as well as in multiple stages of stochastic dynamic programming models. In the paper, we will propose a number of new methods to solve the stochastic optimization problems that occur. In more detail, these problems are analyzed in [20].

\footnotetext{
${ }^{1}$ International Institute for Applied Systems Analysis, Luxenburg, Austria, ermoliev@iiasa.ic.it. ${ }^{2}$ V. M. Glushkov Institute of Cybernetics, National Academy of Sciences of Ukraine, Kyiv, Ukraine, ${ }^{\dagger}$ vladimir.norkin@gmail.com; \$bogdan.norkin@gmail.com. Translated from Kibernetika i Sistemnyi Analiz, No. 1, January-February, 2020, pp. 70-81. Original article submitted March 26, 2019.
} 


\section{THE E-MODEL OF STOCHASTIC PROGRAMMING}

Let us consider the following conceptual model of an insurance company. Let $u \geq 0$ denote an insurance reserve from some feasible set $U, x \in X$ be planned volume of insurance contracts (in money terms) from a feasible set $X, v \geq 0$ be insurance load, $c(x)$ be net premium on insurance contracts (gross premium $(1+v) x$ minus operating costs to keep the contract volume at the level $x$ ), $\xi \geq 0$ be (random) loss ratio of the insurance business (with the distribution function $F(\cdot)$ ), i.e., a random value of insurance claims corresponding to unit premium. In actuarial statistics, $\xi$ is called payout level [21]. Stochastic programming theory considers the so-called two-stage decision-making model. As applied to actuarial modeling, it looks as follows: at the first stage, a deterministic solution $(u, x)$ is made and at the second stage $\xi$ is observed, i.e., the value of insurance claims $\xi x$ becomes known. The objective of the business is to maximize dividends $d$, which can be both deterministic or random, depending on the statement. Let the decision about dividends be made before insurance claims $\xi x$ become finally known. Thus, if company's capital is $u$ at the first stage, then at the second stage it is a random variable

$$
f(u, x, d, \xi)=u+c(x)-d-\xi x,
$$

which can be both positive or negative. In the latter case, insolvency of the company is said to occur. Within the limits of the standard stochastic programming theory, it is assumed that the insolvency problem can be solved by borrowing a capital of the value $(-\min \{0, u+c(x)-d-\xi x\})$ multiplied by insolvency penalty coefficient $q>0$ (it is natural to suppose that $q \geq 1$ ). Then the problem of making a decision about the values of $u, x$, and $d$ is to maximize the total expected income (E-problem):

$$
F(u, x, q)=d+q \mathrm{E}_{\xi} \min \{0, u+c(x)-d-\xi x\} \rightarrow \max _{(u, x) \in W \subseteq U \times X, d \geq 0},
$$

where $\mathrm{E}_{\xi}$ is operator of expectation with respect to $\xi$. If the constraint set $W \subseteq U \times X$ is single-point, i.e., $u$ and $x$ are fixed, then as a matter of fact problem (2) is only related to choosing prepaid dividends $d$. For a fixed parameter $q$ and a concave function $c(x)$, problem (2) is a convex stochastic programming problem and various methods exist for its solution [15-19]. Let $\left(u^{*}, x^{*}, d^{*}\right)$ be the optimal solution of problem (2). For some values of $\xi$, insolvency of the company may take place, $f\left(u^{*}, x^{*}, d^{*}, \xi\right)<0$, or in other words, the probability of insolvency $\operatorname{Pr}\left\{f\left(u^{*}, x^{*}, d^{*}, \xi\right)<0\right\}$ is greater than zero. However, by increasing the penalty coefficient $q$, it is possible to attain desirable reduction of this probability [22].

\section{THE P-MODEL OF STOCHASTIC PROGRAMMING}

There are also other statements of stochastic programming problems, for example, so-called P-problems [17]. Let us use the notation from the previous section and consider the problem

$$
d \rightarrow \max _{(u, x) \in W \subseteq U \times X, d \geq 0}
$$

under the probability constraint

$$
\operatorname{Pr}\{u+c(x)-\xi x-d<0\} \leq \varepsilon,
$$

where $\operatorname{Pr}\{\cdot\}$ is the probability of the event in curly brackets and $\varepsilon$ is the parameter of reliability of the insurance business, $0<\varepsilon<1$. Unlike (2), the model (3), (4) allows explicit control of the insolvency probability but appears nonconvex. Indeed, denote by $F(z)=\operatorname{Pr}\{\xi \leq z\}$ the distribution function of random variable $\xi$. Then

$$
\operatorname{Pr}\{u+c(x)-d-\xi x<0\}=\operatorname{Pr}\{\xi>(u+c(x)-d) / x\}=1-F((u+c(x)-d) / x)
$$

and thus we can rearrange the constraint as

$$
F((u+c(x)-d) / x) \geq 1-\varepsilon .
$$

From here, it can be seen that constraint (5) and hence (4) can be nonconvex, which considerably complicates the solution of problem (3), (4). 


\section{MODELING THE INSURANCE PORTFOLIO}

In insurance, portfolio means the structure of insurance premium with respect to different aspects of insurance. We will describe insurance portfolio by the vector $x \in X$ with components $\left(x_{1}, \ldots, x_{n}\right)$, where $X$ is the set of feasible portfolios, for example, $X=\left\{x \geq 0: \sum_{i=1}^{n} x_{i} \leq w\right\}$, with a fixed nominal cost $w$. Denote by $c\left(x_{i}\right)$ and $\xi_{i}$ net profit and random unprofitability of the $i$ th kind of insurance (for example, in annual expression), $\xi=\left(\xi_{1}, \ldots, \xi_{n}\right)$. Then similarly to (1), for the initial capital $u$ and dividend payouts $d$, random capital at the year end will be

$$
f(u, x, d, \xi)=u+\sum_{i=1}^{n} c_{i}\left(x_{i}\right)-d-\sum_{i=1}^{n} x_{i} \xi_{i} .
$$

Problems of insurance portfolio optimization can be formulated similarly to (2):

or similarly to (3), (4):

$$
F(u, x, q)=d+q \mathrm{E}_{\xi} \min \{0, f(u, x, d, \xi)\} \rightarrow \max _{(u, x) \in W \subseteq U \times X, d \geq 0} ;
$$

$$
\begin{aligned}
& d \rightarrow \max _{(u, x) \in W \subseteq U \times X, d \geq 0}, \\
& \operatorname{Pr}\{f(u, x, d, \xi)<0\} \leq \varepsilon .
\end{aligned}
$$

In the multidimentional case, the probability constraint (8) cannot be expressed in terms of distribution function like in (5); therefore, problem (7), (8) needs special solution techniques to be developed.

\section{REDUCING THE P-MODEL OF STOCHASTIC PROGRAMMING TO PARTIALLY INTEGER OPTIMIZATION PROBLEMS}

If random variable $\xi$ takes only a finite number of values (scenarios) $\left\{\xi_{1}, \ldots, \xi_{k}\right\}$ with probabilities $\left\{p_{1}, \ldots, p_{k}\right\}$, then it is possible to reduce problem (3), (4) to a partially integer programming problem in an equivalent way. Let $z=\left\{z_{1}, \ldots, z_{k}\right\}$ be a set of Boolean variables and $M$ be a sufficiently big constant. Then problem (3), (4) is equivalent to the following partially integer programming problem:

$$
\begin{gathered}
d \rightarrow \max _{(u, x) \in W, d \geq 0, z \in\{0,1\}^{k}} \\
\sum_{i=1}^{k} p_{i} z_{i} \geq 1-\varepsilon, \\
u+c(x)-d-\xi_{i} x \geq-M\left(1-z_{i}\right), \quad i=1, \ldots, k .
\end{gathered}
$$

Here, constraint (10) means that total probability of the scenarios $i$ for which $z_{i}=1$ is greater than or equal to $(1-\varepsilon)$. For $z_{i}=1$, the respective constraint $u+c(x)-d-\xi_{i} x \geq-M\left(1-z_{i}\right)$ will be transformed to the inequality $u+c(x)-d-\xi_{i} x \geq 0$, and for $z_{i}=0$ it becomes the inequality $u+c(x)-d-\xi_{i} x \geq-M$ and since $M$ is large, it holds automatically, i.e., is not limiting. Thus, constraints (10) and (11) in aggregate yield the probability constraint (4). Such an approach to reducing a problem with probability constraint to a partially integer programming problem (for the first time, it was proposed in [23], see also [24]) is applied in [9, 20] to insurance portfolio optimization under a constraint for company's ruin probability.

\section{VECTOR PROBLEM OF STOCHASTIC PROGRAMMING}

The problem of insurance business optimization can be considered as a multicriteria one. For example, instead of problems (2) and (3), (4), it is possible to consider the following two-criteria problems: 


$$
\begin{gathered}
f_{1}(u, x, d)=d \rightarrow \max _{(u, x) \in W, d \geq 0}, \\
f_{2}(u, x, d)=-\mathrm{E}_{\xi} \min \{0, u+c(x)-d-\xi x\} \rightarrow \min _{(u, x) \in W, d \geq 0},
\end{gathered}
$$

where the second criterion $f_{2}(u, x, d)$ means average capital deficit at the time of ruin,

$$
\begin{gathered}
f_{1}(u, x, d)=d \rightarrow \max _{(u, x) \in W, d \geq 0}, \\
f_{3}(u, x, d)=\operatorname{Pr}\{u+c(x)-d-\xi x<0\} \rightarrow \min _{(u, x) \in W, d \geq 0} .
\end{gathered}
$$

Effective boundary of the first problem $(12),(13)$ in the plane "profitability-risk" $\left(f_{1}, f_{2}\right)$ is defined by the function

$$
F_{2}(d)=\min _{(u, x) \in W} f_{2}(u, x, d)=\min _{(u, x) \in W} \mathrm{E}_{\xi} \max \{0, d+\xi x-u-c(x)\} .
$$

If $c(x)$ is a linear or concave function, then (16) is a convex stochastic programming problem [15, 16, 18$]$.

Effective boundary of the second problem $(14),(15)$ in the plane "dividends-ruin probability" $\left(f_{1}, f_{3}\right)$ is defined by the function

$$
F_{3}(d)=\min _{(u, x) \in W} f_{3}(u, x, d)=\min _{(u, x) \in W} \operatorname{Pr}\{u+c(x)-d-\xi x<0\}
$$

This problem is a nonconvex stochastic programming problem. In case of scalar $\xi$ and $x$, we get

$$
F_{3}(d)=\min _{(u, x) \in W}[1-F((u+c(x)-d) / x)]
$$

where $F(\cdot)$ is the distribution function of random variable $\xi$.

If the random variable $\xi$ takes a finite set of values (scenarios) $\left\{\xi_{1}, \ldots, \xi_{n}\right\}$ with probabilities $\left\{p_{1}, \ldots, p_{n}\right\}$, then problem (17) similarly reduces to a partially integer programming problem [24]. Indeed, let us introduce Boolean variables $\left\{z_{1}, \ldots, z_{n}\right\}$ and consider the problem

$$
\begin{gathered}
\sum_{i=1}^{n} p_{i} z_{i} \rightarrow \max _{(u, x) \in W, z_{i} \in\{0,1\}^{n}} \\
u+c(x)-d-\xi_{i} x \geq-M\left(1-z_{i}\right), i=1, \ldots, n,
\end{gathered}
$$

where $M$ is a sufficiently big constant. In this problem, non-ruin probability is maximized; therefore, it is equivalent to problem (17).

\section{THE MODEL OF STOCHASTIC PROGRAMMING WITH DECISIVE STRATEGIES}

Let us consider the case where decision about dividend payout is made after insurance claims $\xi x$ become known. In this case, dividends $d=d(\cdot)$ can be an arbitrary measurable function of company's capital $u+c(x)-\xi x$. Then the problem (3), (4) becomes

$$
\mathrm{E}_{\xi} d(u+c(x)-\xi x) \rightarrow \max _{(u, x) \in W, d(\cdot) \geq 0}
$$

under the probability constraint

$$
\operatorname{Pr}\{u+c(x)-\xi x-d(u+c(x)-\xi x)<0\} \leq \varepsilon,
$$


where optimization is performed not only with respect to the variables $u$ and $x$ but also with respect to all (Borel) measurable functions $d(\cdot) \geq 0$. This problem is infinite-dimensional; to solve it, it is necessary to look over all possible measurable functions $d(\cdot)$ together with all feasible values $u$ and $x$. One of the possible approaches to its approximate solution is to represent the parametric form of function $d(\cdot)$. For example, it is possible to be restricted to so-called threshold strategies $d(\cdot, y)=\max \{0, \cdot-y\}$, where $y$ is the parameter of threshold value. The choice of the threshold strategy of dividend payout means that if company's capital is less than a threshold, dividends are not subtracted from the capital, and if the capital is greater than the threshold, the part of the capital exceeding the threshold is subtracted as the dividends. Other kinds of dividend strategies, generally dependent on the finitedimensional vector parameter $y$, are also possible. When function $d(\cdot, y)$ is substituted into the problem (18), (19), the problem is transformed into the finite-dimensional problem

$$
\mathrm{E}_{\xi} d(u+c(x)-\xi x, y)=\mathrm{E} \xi \max \{0,(u+c(x)-\xi x-y)\} \rightarrow \max _{(u, x) \in W, y \geq 0}
$$

under the probability constraint

$$
\operatorname{Pr}\{u+c(x)-\xi x-\max \{0,(u+c(x)-\xi x-y)\}<0\} \leq \varepsilon
$$

and can be solved by direct-search (evolutionary) algorithms. Vector problems (12)-(15) can be re-formulated in the same way. Such approach to solution of complex dynamic problems of stochastic optimal control of the dividend strategy of insurance company is applied in [25].

\section{DYNAMIC MODELS OF STOCHASTIC PROGRAMMING}

Let reserves of the insurance company $X^{t}$ vary with (discrete) time $t=0,1, \ldots$ according to the following relation:

$$
X^{t+1}=X^{t}+c(x)-d\left(X^{t}, y\right)-\xi^{t} x, \quad X^{0}=u, \quad t=0,1, \ldots
$$

Here, $x$ is nominal value of the insurance portfolio; $c(x)$ is deterministic inflows of premiums per unit time from the insurance portfolio $x ; d\left(X^{t}, y\right) \in\left[0, X^{t}\right]$ is a dividend strategy as a function of the current capital and parameter $y$; $\left\{\xi^{t}, t=0,1, \ldots\right\}$ are independent equally distributed (like some random variable $\xi$ with the distribution function $F$ ) observations of insurance payouts. Process (22) is called a risk process. By a ruin (or insolvency) event of a risk process, we will understand implementations of process (22) such that $X^{t}<0$ for some $t>0$. Denote by $\varphi^{t}(u)$ probability of non-ruin of process (22) in $t$ steps for the initial capital $X^{0}=u$ :

$$
\varphi^{t}(u)=\operatorname{Pr}\left\{X^{k} \geq 0,0 \leq k \leq t, X^{0}=u\right\},
$$

where $\operatorname{Pr}\{\cdot\}$ is probability of the event in curly brackets. Function $\varphi$ depends not only on $u$ but also on the other parameters $(x, y)$ of process (22). However, in the given context, this dependence is not specified explicitly. The sequence of functions $\left\{\varphi^{t}(\cdot), t=0,1, \ldots\right\}$ satisfies the integral relations

$$
\begin{gathered}
\varphi^{1}(u)=\operatorname{Pr}\{u+c(x)-d(u)-\xi x \geq 0\} \\
=\operatorname{Pr}\{\xi \leq(u+c(x)-d(u)) / x\}=F((u+c(x)-d(u)) / x), \\
\varphi^{t+1}(u)=\mathrm{E}_{\xi} \varphi^{t}(u+c(x)-d(u)-\xi x), \quad t=1,2, \ldots,
\end{gathered}
$$

where $\mathrm{E}_{\xi}$ is the operator of expectation (Lebesgue integral with respect to the measure induced by the random variable $\xi), F(z)=\operatorname{Pr}\{\xi \leq z\}$; by definition, $\varphi^{t}(u)=0$ for $u<0$ for all $t$. 


\section{INTEGRAL EQUATIONS OF ACTUARIAL MATHEMATICS}

For a fixed $x$, the probability of non-ruin of process $(22)$ on the infinite time interval

$$
\varphi(u)=\operatorname{Pr}\left\{X^{t} \geq 0 \quad \forall t \geq 0, X^{0}=u\right\}
$$

satisfies the (integral) equation

$$
\varphi(u)=\mathrm{E}_{\xi} \varphi(u+c(x)-d(u)-\xi x)=\int_{\{\eta: u+c(x)-d(u)-\eta x \geq 0\}} \varphi(u+c(x)-d(u)-\eta x) d F(\eta),
$$

where by definition $\varphi(u)=0$ for $u<0$. This is a linear integral equation that always has a zero solution. However, of interest are conditions whereby there exists a nondecreasing solution $\varphi(\cdot)$ such that

$$
0 \leq \varphi(u) \leq 1, \lim _{\{u \rightarrow+\infty\}} \varphi(u)=1 .
$$

The studies $[20,26]$ analyze the properties of such equations and conditions of the existence and uniqueness of such solution. As a matter of fact, relations (23) and (24) determine the method of successive approximations for solution of the integral equation (25).

Note that the operator $A \varphi(u)=\mathrm{E}_{\xi} \varphi(u+c(x)-d(u)-\xi x)$ on the right-hand side of (25) is non-expanding with respect to the sup-norm:

$$
\begin{gathered}
|| A \varphi_{1}-A \varphi_{2}||=\sup _{u \geq 0}\left|A \varphi_{1}(u, x)-A \varphi_{2}(u, x)\right| \\
=\sup _{u \geq 0}\left|\mathrm{E}_{\xi} \varphi_{1}(u+c(x)-d(u)-\xi x, x)-\mathrm{E}_{\xi} \varphi_{2}(u+c(x)-d(u)-\xi x, x)\right| \\
\leq \mathrm{E}_{\xi} \sup _{u \geq 0}\left|\varphi_{1}(u+c(x)-d(u)-\xi x, x)-\varphi_{2}(u+c(x)-d(u)-\xi x, x)\right| \\
\leq \mathrm{E}_{\xi} \sup _{y \geq 0}\left|\varphi_{1}(y, x)-\varphi_{2}(y, x)\right|=\mathrm{E}_{\xi}|| \varphi_{1}-\varphi_{2}||=|| \varphi_{1}-\varphi_{2} \| .
\end{gathered}
$$

However, this operator is not contracting; therefore, existence and uniqueness of the solution of Eq. (25), as well as convergence of the method of successive approximations (23), (24), cannot be deduced from the principle of contracting mappings.

\section{NECESSARY AND SUFFICIENT CONDITIONS FOR THE EXISTENCE AND UNIQUENESS OF THE SOLUTIONS OF INTEGRAL EQUATIONS OF ACTUARIAL MATHEMATICS}

Let $A \varphi(u)=\mathrm{E}_{\xi} \varphi(u+c(x)-d-\xi x)$ be the operator on the right-hand side of (25), where $x$ and $d$ are fixed parameters. Assume that the random variable $\xi$ is bounded, $\xi \leq m$ with probability one. Let us find function $\varphi_{*}(u)$ such that $A \varphi_{*}(u) \geq \varphi_{*}(u)$ has the form $\varphi_{*}(u)=\max \left\{0,1-e^{-L\left(u-u_{*}\right)}\right\}$, where $L$ and $u_{*}$ are the unknown constants. The following estimates are true:

$$
A \varphi_{*}(u) \geq \int_{0}^{(u+c(x)-d) / x}\left(1-e^{-L\left(u-u_{*}+c(x)-d-\eta x\right)}\right) d F(\eta)
$$




$$
\begin{gathered}
=F((u+c(x)-d) / x)-e^{-L\left(u-u_{*}\right)} \int_{0}^{(u+c(x)-d) / x} e^{L(x \eta+d-c(x))} d F(\eta) \\
\geq F((u+c(x)-d) / x)-e^{-L\left(u-u_{*}\right)} \int_{0}^{+\infty} e^{L(x \eta+d-c(x))} d F(\eta) .
\end{gathered}
$$

Let us find $u_{*}$ and $L$ such that the following conditions are satisfied:

$$
\left(u_{*}+c(x)-d\right) / x \geq m, \int_{0}^{+\infty} e^{L(x \eta+d-c(x))} d F(\eta) \leq 1,
$$

then from (27) for $u \geq u_{*}$ we obtain $A \varphi_{*}(u) \geq 1-e^{-L\left(u-u_{*}\right)}$ and thus for all $u \geq 0$ we get $A \varphi_{*}(u)$ $\geq \max \left\{0,1-e^{-L\left(u-u_{*}\right)}\right\}=\varphi_{*}(u)$. This condition is necessary and sufficient for the existence of the solution of problem (25), (26) [26].

Applying the operator $A$ to the step function $\mathbf{1}(u)=\left\{\begin{array}{ll}1, & u \geq 0, \\ 0, & u<0,\end{array}\right.$ yields

$$
A \mathbf{1}(u)=\int_{0}^{(u+c(x)-d) / x} d F(\eta)=F((u+c(x)-d) / x) .
$$

If $F(y)<1$ for all $y$, then $A \mathbf{1}(u)<1$ for all $u \geq 0$. This condition is sufficient for the uniqueness of the solution of problem (25), (26). The necessary conditions for the uniqueness of the solution (of this kind) are presented in [26].

\section{DISCOUNTED PARAMETERS OF OPERATION}

For process (22), let us consider a number of additive functionals of utility and risk. Let at each step process (22) be characterized by the parameter $r(\cdot, \xi)$, then we will define the functional, additive in the trajectory of the process

$$
V(u, x, y)=\mathrm{E} \sum_{t=0}^{\tau-1} \gamma^{t} r\left(X^{t}, \xi^{t}\right),
$$

where $0<\gamma<1$, the expectation $\mathrm{E}$ can be calculated over all possible trajectories of the process, and $\tau$ is a random instant of time of ruin of the process, i.e.,

$$
\tau=\sup \left\{t: \min _{0 \leq k<t} X^{k} \geq 0\right\} .
$$

Note that function $V(u, x, y)$ depends on the parameters $(u, x, y)$ of process (22) but it does not contain any operations of supremum or infimum unlike the standard Bellman optimality function. If function $r(\cdot)$ grows no faster than linear one, then function $V(\cdot, x, y)$ satisfies the equation [27]

$$
V(u, x, y)=\mathrm{E}_{\xi} r(u, \xi)+\gamma \mathrm{E}_{\xi} V(u+c(x)-d(u, y)-x \xi, x, y),
$$

where $V(u, x, y)=0$ for $u<0$. Note that unlike the standard Bellman equation, the right-hand side of (28) does not contain any operations of supremum or infimum.

If $r(\cdot)$ is upper semicontinuous and $c(x)$ and $d(u, y)$ are continuous in their arguments, then $V(u, x, y)$ is upper semicontinuous and for each fixed pair $(x, y)$, its values can be found by the method of successive approximations [27]:

$$
\begin{gathered}
V^{k+1}(u, x, y)=r(u)+\gamma \mathrm{E}_{\xi} V^{k}(u+c(x)-d(u, y)-\xi x, x, y), \\
V^{0}(u, x, y)=0, k=0,1, \ldots
\end{gathered}
$$


For example, if

$$
r_{0}\left(X^{t}\right)=\left\{\begin{array}{cc}
d\left(X^{t}, y\right), & X^{t} \geq 0, \\
0, & X^{t}<0,
\end{array}\right.
$$

then average discounted dividends till the moment of ruin can be expressed as

$$
V_{0}(u, x, y)=\mathrm{E} \sum_{t=0}^{\tau-1} \gamma^{t} d\left(X^{t}, y\right)
$$

and function $V_{0}(\cdot, x, y)$ satisfies the equation

$$
V_{0}(u, x, y)=d(u, y)+\gamma \mathrm{E}_{\xi} V_{0}(u+c(x)-d(u, y)-x \xi, x, y) .
$$

If

$$
r_{1}\left(X^{t}\right)= \begin{cases}1, & X^{t} \geq 0, \\ 0, & X^{t}<0,\end{cases}
$$

then average discounted lifetime of the process

$$
V_{1}(u, x, y)=\mathrm{E} \sum_{t=0}^{\tau-1} \gamma^{t}
$$

satisfies the equation

$$
V_{1}(u, x, y)=1+\gamma \mathrm{E}_{\xi} V_{1}(u+c(x)-d(u, y)-x \xi, x, y) .
$$

If

$$
r_{2}\left(X^{t}, \xi^{t}\right)=\left\{\begin{array}{cc}
\mathbf{1}_{\left\{X^{t}+c(x)-d\left(X^{t}, y\right)-\xi^{t} x<0\right\}}, & X^{t} \geq 0, \\
0, & X^{t}<0,
\end{array}\right.
$$

then (discounted) ruin probability

$$
V_{2}(u, x, y)=\mathrm{E} \sum_{t=0}^{\tau-1} \gamma^{t} \mathbf{1}_{\left\{X^{t}+c(x)-d\left(X^{t}, y\right)-\xi^{t} x<0\right\}}
$$

satisfies the equation

$$
\begin{gathered}
V_{2}(u, x, y)=\mathrm{E}_{\xi} \mathbf{1}_{\{u+c(x)-d(u, y)-\xi x<0\}}+\gamma \mathrm{E}_{\xi} V_{2}(u+c(x)-d(u, y)-\xi x, x, y) \\
=\left(1-F((u+c(x)-d(u, y)) / x)+\gamma \mathrm{E}_{\xi} V_{2}(u+c(x)-d(u, y)-\xi x, x, y),\right.
\end{gathered}
$$

where $F(z)=\operatorname{Pr}\{\xi \leq z\}$.

\section{OPTIMAL VECTOR CONTROL OF THE RISK PROCESS}

The problem of optimal vector control of the risk process (22) is to find non-dominating values of the vector parameter $\overrightarrow{\mathbf{V}}(u, x, y)=\left\{V_{i}(u, x, y), i=0,1, \ldots\right\}$ :

$$
\overrightarrow{\mathbf{V}}(u, x, y) \rightarrow \operatorname{extr}_{(u, x) \in W, y \in Y},
$$

as well as respective Pareto-optimal values of parameters $(u, x, y)$, where $u$ denotes initial value of the risk process (22), $x$ describes the structure of insurance portfolio, and parameter $y$ determines the choice of dividend strategy. For example, from heuristic reasons, for numerical approximation of Pareto-optimal boundary, so-called threshold-proportional strategies of control of dividend deductions is considered, i.e., $d(x, y)=y_{1} \max \left\{0, x-y_{2}\right\}$, $y=\left(y_{1}, y_{2}\right), y_{1} \in[0,1], y_{2} \geq 0$.

The complexity of this problem is that, first, the parameters $V_{i}(u, x, y)$ are not known in explicit form and are solutions of respective integral equations; second, these parameters can be nonconvex functions; third, respective Pareto-optimal set may have rather complicated structure. 
The values of functions $V_{i}(u, x, y)$ can be found by the method of successive approximations (29) or by the method of statistical trials, in particular, by their parallel versions.

For small dimensions of the vector of parameters $(u, x, y)$, problem (30) can be solved approximately by means of discrete approximation of sets $W$ and $Y$ by finite sets $W^{N}$ and $Y^{N}$ and solutions of the discrete problem of vector optimization

$$
\overrightarrow{\mathbf{V}}(u, x, y) \rightarrow \operatorname{extr}_{(u, x) \in W^{N}, y \in Y^{N}} .
$$

Such method of solution of vector optimization problems is considered in [28] and its convergence is analyzed in [29-31].

\section{CONCLUSIONS}

In the paper, we have reviewed the conceptual optimization models of insurance activity based on the paradigm of stochastic programming. Operation of insurance companies can be described by a random process of evolution of capital in discrete time. The main random factor of evolution is insurance claims. The main characteristics of operation of companies are indicators of average efficiency (for example, of expected profitability) and risk indicators (for example, insolvency probability or the value of the necessary borrowed capital). We have considered one-, two-, and multi-stage models, as well as multicriteria statements. The complexity of optimization problems that arise is caused by not only random parameters but also non-convexity of the problems. Moreover, insurance company operation indicators are not known in explicit form and are either multidimensional integrals of the type of expectation or solutions of integral equations of actuarial mathematics. These circumstances transform the problem of optimization of insurance activity into a complicated computing problem. In the paper, we have proposed approaches to solution of these problems on the basis of the methods of stochastic programming, integer programming, multicriteria optimization, and dynamic programming.

\section{REFERENCES}

1. R. Kaas, M. Goovaerts, J. Dhaene, and M. Denuit, Modern Actuarial Risk Theory, Kluwer Academic Publ., Boston (2001).

2. N. L. Bowers, H. U. Gerber, J. C. Hickman, D. A. Jones, and C. J. Nesbitt, Actuarial Mathematics, Society of Actuaries, Chicago (1997).

3. B. de Finetti, "Su un'impostazione alternativa della teoria collettiva del rischio," in: Trans. 15th Intern. Congress of Actuaries, Vol. 2, Actuarial Society of America, New York (1957), pp. 433-443.

4. R. E. Beard, T. Pentikainen, and E. Pesonen, Risk Theory. The Stochastic Basis of Insurance, Chapman and Hall, London-New York (1984).

5. A. N. Nakonechnyi, "Optimization of risk processes," Cybern. Syst. Analysis, Vol. 32, No. 5, 641-646 (1996).

6. G. I. Lyubchenko and A. N. Nakonechnyi, "Optimization methods for compound Poisson risk processes," Cybern. Syst. Analysis, Vol. 34, No. 2, 230-237 (1998).

7. E. Bayraktar and V. Young, "Maximizing utility of consumption subject to a constraint on the probability of lifetime ruin,” Finance and Research Letters, Vol. 5, Iss. 4, 204-212 (2008). DOI: 10.1016/j.frl.2008.08.002.

8. H. Schmidli, Stochastic Control in Insurance, Springer-Verlag, London (2008).

9. B. V. Norkin, "On optimization of insurance portfolio," Applied Statistics. Actuarial and Financial Mathematics, No. 1-2, 197-203 (2011). URL: https://www.researchgate.net/publication/260869967_On_optimization_of_ insurance portfolio.

10. B. V. Norkin, "Mathematical models for insurance business optimization," Cybern. Syst. Analysis, Vol. 47, No. 1, 117-133 (2011).

11. A. N. Gromov, "Optimal reinsurance and investment strategy," Vestn. Mosk. Univer., Ser. 1, Mathematics. Mechanics, Issue 2, 6-12 (2013). URL: https://cyberleninka.ru/article/v/optimalnaya-strategiya-perestrahovaniyai-investirovaniya. 
12. S. Asmussen and H. Albrecher, Ruin Probabilities, World Scientific, London (2010).

13. V. Yu. Korolev, B. E. Bening, and S. Ya. Shorgin, The Mathematical Fundamentals of Risk Theory [in Russian], Fizmatlit, Moscow (2011).

14. M. M. Leonenko, Yu. S. Mishura, Ya. M. Parkhomenko, and M. J. Yadrenko, Probability-Theoretic and Statistical Methods in Econometry and Financial Mathematics [in Ukrainian], Informtekhnika, Kyiv (1995).

15. A. Shapiro, D. Dentcheva, and A. Ruszczynski, Lectures on Stochastic Programming: Modeling and Theory, SIAM, Philadelphia (2009).

16. Yu. M. Ermoliev, Methods of Stochastic Programming [in Russian], Nauka, Moscow (1976).

17. Yu. S. Kan and A. N. Kibzun, Stochastic Programming Problems with Probability Criteria [in Russian], Fizmatlit, Moscow (2009).

18. D. B. Yudin, Problems and Methods of Stochastic Programming [in Russian], Sov. Radio, Moscow (1979).

19. W. B. Powell, "Clearing the jungle of stochastic optimization," in: INFORMS Tutorials in Operations Research, Published online: 27 Oct. (2014), pp. 109-137. http://dx.doi.org/10.1287/educ.2014.0128.

20. B. V. Norkin, Numerical Methods to Solve Stochastic Optimization Problems in Actuarial Mathematics, Author's Abstracts of Ph.D. Theses, V. M. Glushkov Institute of Cybernetics, NAS of Ukraine (2015).

21. Forinsurer. URL: https://forinsurer.com/ratings/nonlife.

22. Y. M. Ermoliev, T. Y. Ermolieva, G. MacDonald, and V. I. Norkin, "Stochastic optimization of insurance portfolios for managing exposure to catastrophic risks," Ann. Oper. Res., Vol. 99, 207-225 (2000). DOI: https://doi.org/10.1023/A:1019244405392.

23. A. Ruszczynki, "Probabilistic programming with discrete distributions and precedence constrained knapsack polyhedral," Math. Program., Vol. 93, 195-215 (2002). https://doi.org/10.1007/s10107-002-0337-7.

24. V. I. Norkin and S. V. Boiko, "Safety-first portfolio selection," Cybern. Syst. Analysis, Vol. 48, No. 2, 180-191 (2012).

25. B. V. Norkin, "Random search of multicriterion optimum in insurance," in: Proc. 4th Intern. Scientific Conf. of Students and Young Scientists "Theoretical and Applied Aspects of Cybernetics" (TAAC-2011. Kyiv, Nov. 24-28, 2014), Bukrek, Kyiv (2014), pp. 176-187. URL: https://www.researchgate.net/publication/301764924_Random_ Search_of_Multicriterion_Optimum_in_Insurance.

26. B. V. Norkin, "Necessary and sufficient conditions of existence and uniqueness of solutions to integral equations of actuarial mathematics," Cybern. Syst. Analysis, Vol. 42, No. 5, 743-749 (2006).

27. B. V. Norkin, "Stochastic optimal control of risk processes with Lipschitz payoff functions," Cybern. Syst. Analysis, Vol. 50, No. 5, 774-787 (2014).

28. I. M. Sobol' and R. B. Statnikov, Choosing Optimal Parameters in Multi-Criteria Problems [in Russian], Drofa, Moscow (2006).

29. B. V. Norkin, "Sample approximations of multiobjective stochastic optimization problems," Optimization-online, Nov. (2014). URL: http://www.optimization-online.org/DB HTML/2014/11/4655.html.

30. B. V. Norkin, "Statistical approximation of multicriteria problems of stochastic programming," Dopov. Nac. Akad. Nauk Ukr., No. 4, 35-41 (2015). https://doi.org/10.15407/dopovidi2015.04.035.

31. B. V. Norkin, "On the approximation of vector optimization problems," Kibernetika i Vych. Tekhnika, Issue 179, 35-42 (2015). URL: http://dspace.nbuv.gov.ua/bitstream/handle/123456789/86145/03-Norkin.pdf?sequence=1. 